\title{
Depth of field simulation for still digital images using a 3D camera
}

\author{
Omar Alejandro Rodríguez Rosas
}

Asistente de Investigación en Universidad de Guadalajara Guadalajara, México omar.alejandro.rodriguez@gmail.com

\begin{abstract}
In a world where digital photography is almost ubiquitous, the size of image capturing devices and their lenses limit their capabilities to achieve shallower depths of field for aesthetic purposes. This work proposes a novel approach to simulate this effect using the color and depth images from a 3D camera. Comparative tests yielded results similar to those of a regular lens.
\end{abstract}

Keywords: bokeh; depth of field; simulation.

Profundidad de simulación de campo para imágenes fijas digitales utilizando una cámara 3D

Resumen: En un mundo donde la fotografía digital es casi omnipresente, el tamaño de los dispositivos de captura de imagen y sus lentes limitan sus capacidades para alcanzar profundidades menores de campo para fines estéticos. Este trabajo propone un enfoque novedoso para simular este efecto usando el color e imágenes profundas de una cámara 3D. Las pruebas comparativas dieron resultados similares a los de una lente regular.

Palabras clave: bokeh; profundidad de campo; simulación. 


\section{Introduction}

Since the release of the first commercial camera in the early 1990s, digital photography has stopped being perceived as a luxury reserved for the wealthiest and progressively became inherent to our daily lives. The new semiconductor technologies and manufacturing techniques allow vendors to attach digital cameras to a huge variety of appliances from mobile phones and tablets to medical equipment and wearable devices by progressively reducing their cost and physical dimensions.

Nevertheless, these reductions have compromised to some extent the quality of the captured images: by allowing them to adjust to the increasingly tighter size constraints of the market, some features from high-end cameras like flexible depths of field and the lens bokeh need to be sacrificed.

Depth of field, in optics, is defined as the distance at which objects in front or behind the focal plane appear acceptably sharp. Other points in the scene outside this area render themselves as blurry spots shaped as the camera's diaphragm whose diameter contract gradually as its distance approaches the focal plane (see figure 1). The maximum diameter of one of these spots that is indistinguishable from a focused point is called maximum permissible circle of confusion or simply circle of confusion. The appearance of these unfocused areas, that is, how pleasant or unpleasant they are, will depend on a number of factors including size, shape and number of blades of the camera's diaphragm and optical aberrations of the lens. The Japanese term bokeh is often employed as a subjective measure to describe the aesthetic quality of the out-of-focus areas in the final picture (Buhler \& Dan, 2002). 


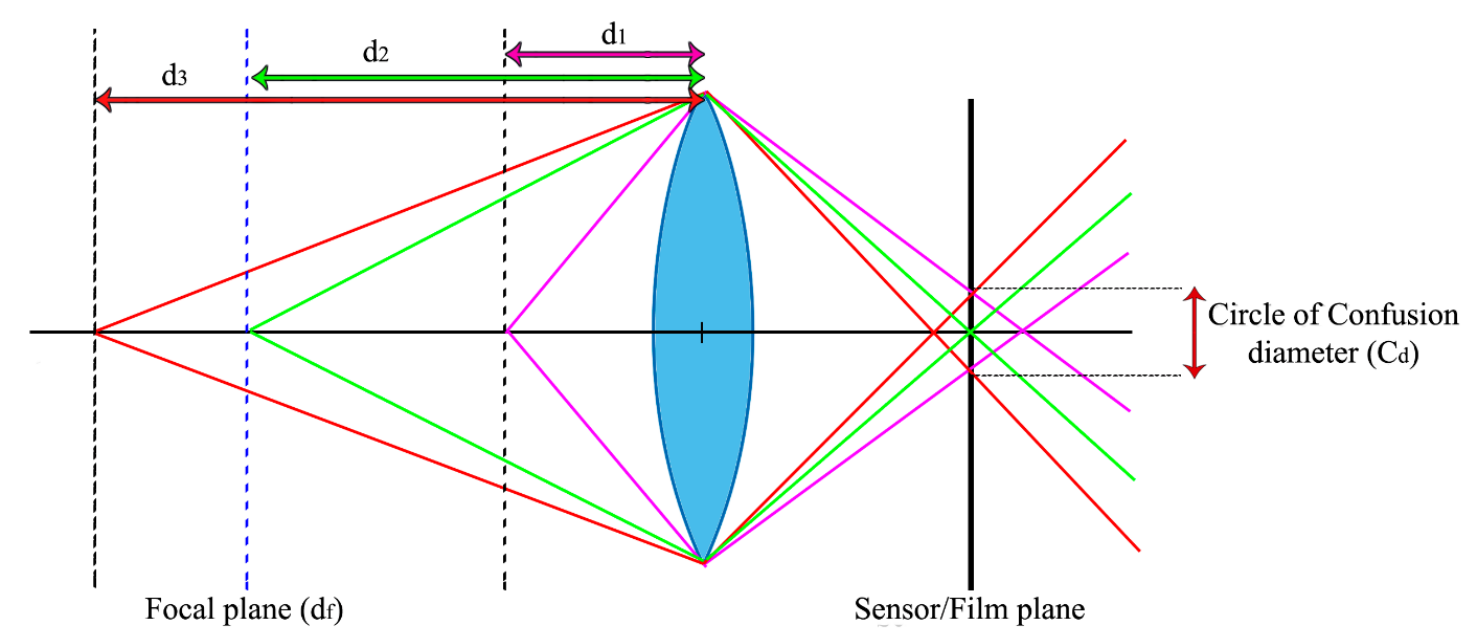

Figure 1. Schematic view of the Circle of Confusion physics.

\section{Depth of field in modern small devices}

A shallower depth of field (which means a bigger circle of confusion) is often a desired behavior since it provides emphasis to certain subjects in a picture, but implies the use of bigger aperture values and focal lengths. Given these requirements, it's not hard to understand why the effect is often disregarded in portable devices such as tablets and cellphones $(Z, 2014)$ where physical size and final price constraints for the finished product and its components are an important design factor.

\section{Current solutions}

Depth of field simulation is not a new technique and it is, in fact, quite common in areas such as 3D rendering where a distance dependent blur is achieved through Gaussian filtering or post processing methods as circle of confusion physics simulation (Rigger, Tatarchuk, \& Isidoro, 2003). Other real-time optimized techniques in the graphics industry suggest to render two separate 
versions of each frame: one without any visible Depth of Field and a blurred representation of the same image. The data from the z-buffer, which contains depth information for each pixel, is then interpreted as an alpha channel to blend the two separate frames reducing the sharpness and color saturation in the outof-focus portions of the scene (U.S. Patent No. 7081892, 2002). Although realistic, efficient and well suited for 3D rendering, due to the lack of depth data, these techniques are not an option for standard digital photography. The problem is then reduced to the acquisition of each point's 3D position.

One way to obtain depth information from digital photographs is the use of light field cameras. This kind of device utilize an array of microscopic lenses placed between the main lens and the photo sensor to sample information about the light field of the image, containing the direction of the light rays passing through each point, which is typically employed to reconstruct the picture using ray-tracing techniques to simulate its imaging plane $(\mathrm{Ng}, 2006)$. Nevertheless, this technology is not yet widely adopted, nor available for portable devices.

To address both depth data acquisition from 2D digital images and the representation of distance dependent blurring, the Google Camera App for Android 4.4 provides the "Lens blur" mode which enables users to take pictures using simulated shallow depths of field, similar to those of SLR cameras. This application relies on the approximation of a 3D map of the scene based on a series of consecutive photographs. The initial images are obtained from a continuous stream whose capture is controlled by the user as an upward sweep. The resulting pictures will then represent the scene from different elevation angles. Using Structure-from-Motion (SfM), Bundle Adjustment and Multi-View Stereo (MVS) algorithms, the estimated 3D positions of each point in the image can be triangulated and the resulting map employed to render the appropriate amount of blur for each pixel according to its depth using a Thin Lens approximation (Hernández, 2014). This method, along with similar technologies from vendors as HTC or Samsung, are indeed precise but require a timeconsuming preprocessing in order to construct the 3D map. 
As an alternative, some relatively inexpensive 3D cameras like Microsoft Kinect or Creative Senz3d can provide a three-dimensional map of the scene at up to 30 frames per second, but since they are primarily targeted for PC's, up until recently, developments for portable devices based on these technologies were not a realistic option. Fortunately, after the announcements by some vendors regarding the inclusion of similar technologies in tablets and laptops in the near future (Tibken, 2013), and the launching of Occipital's Structure sensor (a depth sensor for $\mathrm{iPad}$ ) earlier this year, the possibility of practical solutions utilizing this kind of device sounds more and more feasible.

Considering that, the following sections propose a theoretical methodology to achieve depth of field simulation and an implementation on currently available hardware that should be easily portable to other mobile technologies as soon as they are available.

\section{Solution proposal}

This proposal for depth of field simulation uses both the color and depth feeds from a 3D camera. As portrayed in figure 2, a summary of the required steps is:

1. Read depth and color frames from the camera.

2. Create a copy of the color frame

3. Apply a blur function to the color frame copy.

4. If necessary, apply a rectification function to map depth pixels to color space.

5. Use a pixel-weight function to translate depth data into alpha channel values.

6. Blend the two color frames (the original and the blurred one) using the previously calculated alpha values. 


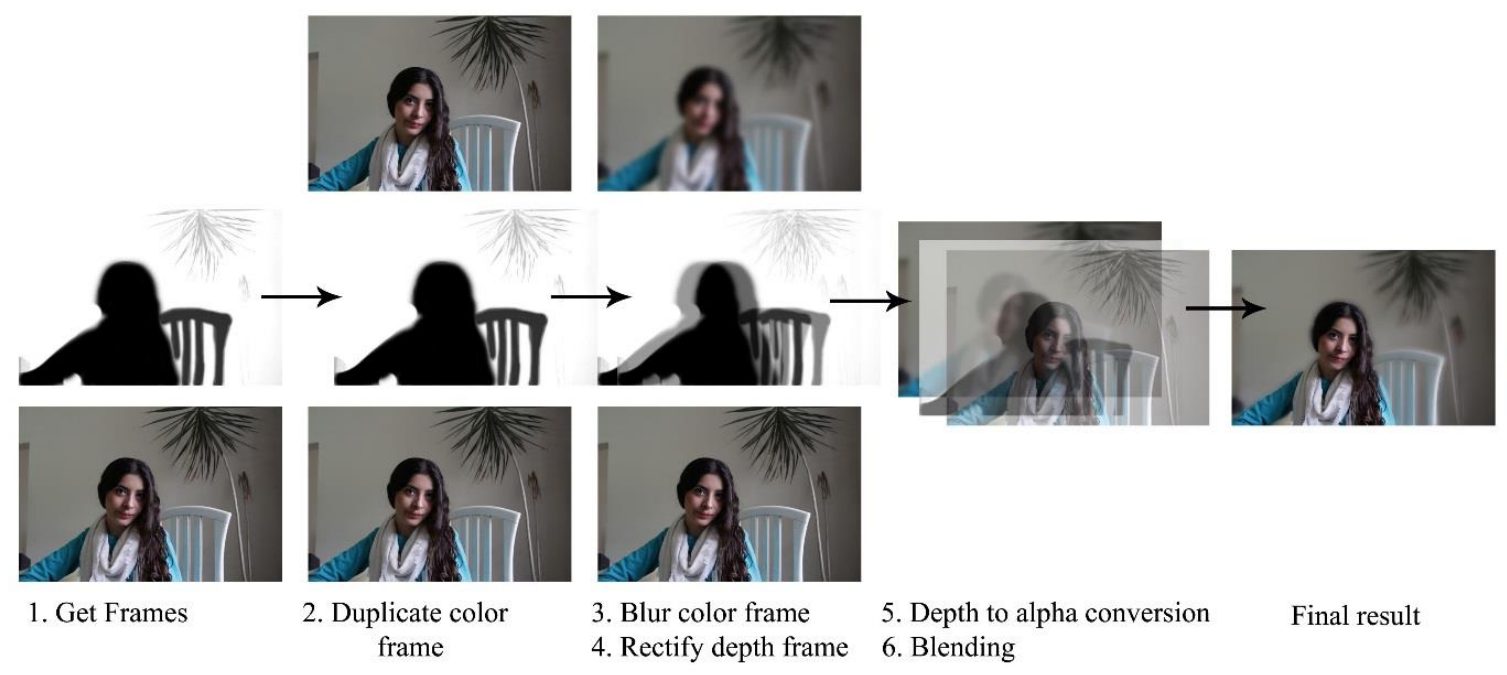

Figure 2. The Depth of field simulation process.

Some of these steps are further explained next.

\subsection{Blur function}

During this stage, blurring will be applied the entire duplicated color frame. The intensity of the effect should be the expected for the farthest points from the focal plane. In the absence of a physical lens to render its optical aberrations to the sensor, the quality of the bokeh will be determined by this step of the process, hence the importance of the method selection. To take an appropriate decision, it is useful to consider that while standard point sampling techniques have uniform density distributions, real lenses tend to display a distinct behavior at different planes, which can be achieved by implementing arbitrary probability density functions to jitter the sampling points amongst those planes (Buhler \& Dan, 2002). The final selection will depend on the particular implementation requirements such as performance, available hardware, accuracy and other considerations regarding the quality and computational cost trade-off. Good candidates for this function are Separable Gaussian Blur, Circle of confusion simulation (Rigger, Tatarchuk, \& Isidoro, 2003), Optical Aberration-based models (Wu, Zheng, Hu, Wang, \& Zhang, 2010), Box blur and FFT-based models. 


\subsection{Rectification function}

Since 3D cameras generally use two lenses at slightly different resolutions and distances from each other, a noticeable offset between the color and depth frames may exist. To achieve realistic results, it is necessary to map each depth pixel information to its corresponding color space representation.

This operation can be performed by standard Epipolar-geometry-based rectification methods (such as those designed for stereoscopic cameras calibration) which, although out of the scope of this article, are implemented in several API's for computer vision including OpenCV and the Microsoft Kinect SDK.

\subsection{Pixel-weight function}

Using this function we translate depth values from the 3D camera's sensor to transparency alpha values which will be applied to blend the blurred version of the color image in order to make objects whose circles of confusion are supposed to be smaller fully transparent and vice versa. For biconvex lenses (commonly used for photography) the relationship between the diameter of the circle of confusion and the distance from the subject to the focal length is described by equation 1:

$$
C_{d}=A\left(\left|d-d_{f}\right|\right) / d
$$

Where $C_{d}$ is the diameter of the circle of confusion, $A$ the aperture value, $d$ the distance of a given object from the lens and $d_{f}$ the distance from the lens to the focal plane (see figure 1). The circle of confusion diameter as a function of the distance from a subject for a given aperture value and focal plane is depicted in figure 3. 

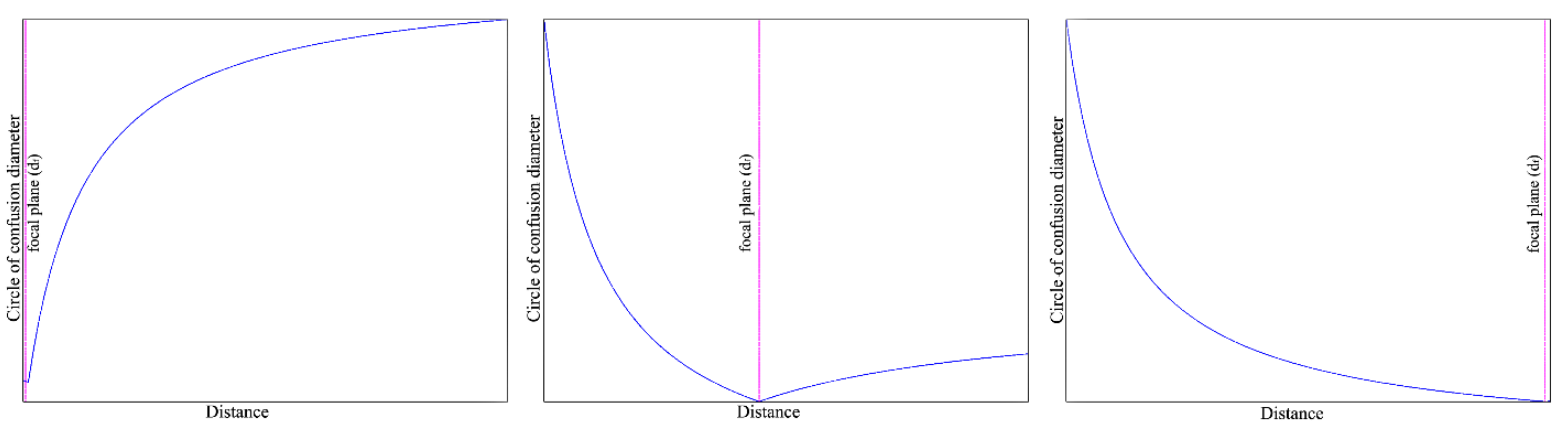

Figure 3. Equation 1 behavior for different focal planes: Near the lens (left), at a medium distance (center) and far from the lens (right)

Other functions, particularly those of the Gaussian distribution family (see equation 2) can also provide interesting results as depicted in figure 4.

$$
f\left(d, d_{f}, \sigma\right)=1-e^{-\frac{\left(d-d_{f}\right)^{2}}{2 \sigma^{2}}}
$$

Where , $d_{f} \in R, 0 \leq d \leq 1$ and $0 \leq d_{f} \leq 1$, representing the ratio of such distance over the max distance range of the camera.
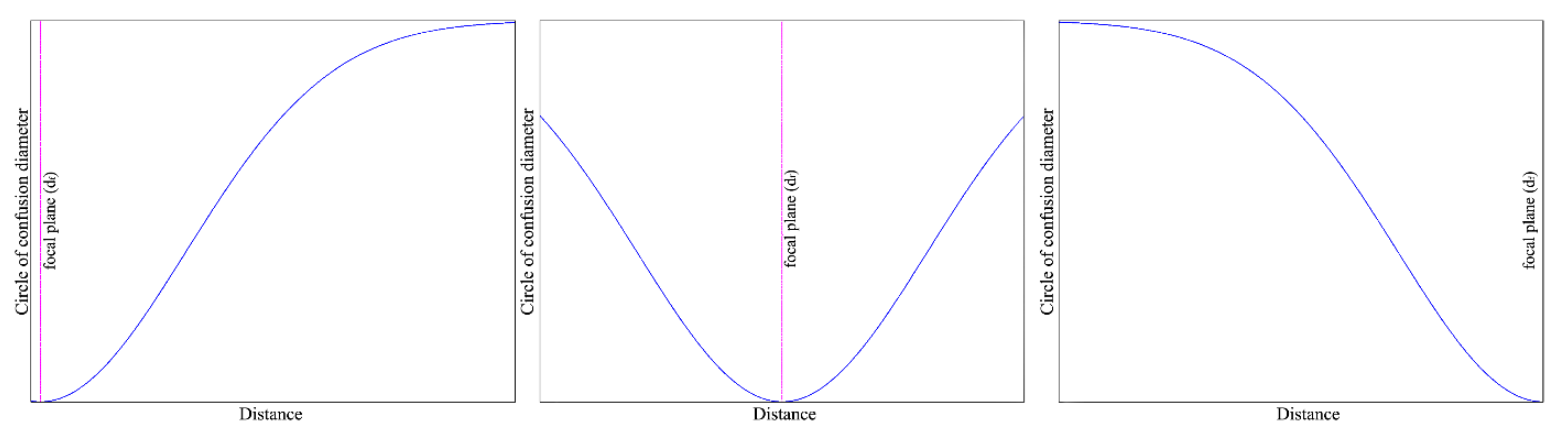

Figure 4. Equation 2 behavior for different focal planes: Near the lens (left), at a medium distance (center) and far from the lens (right)

\subsection{Blending function}

Once the blur function has been applied to the copy of the color image and the alpha values have been calculated, a linear blending function will combine the two color frames into one according to the weight value defined from the depth 
data. To do so, for each pixel, the final color value is calculated using equation 3:

$$
O_{R G B}^{\prime}=B_{R G B} \alpha+O_{R G B}(1-\alpha)
$$

which is a simplification of the general linear blending equation assuming a totally opaque background where $\mathrm{O}_{\mathrm{RGB}}$ is a pixel from the blended output color image, $B_{R G B}$ is the blurred version of the original color image, $\alpha$ is an alpha value such that $0 \leq \alpha \leq 1$ and $\mathrm{O}_{\mathrm{RGB}}$ is a pixel from the original color frame.

\section{Implementation}

For this paper, an implementation using Microsoft's Kinect for Windows and its SDK has been coded. The color and depth information are retrieved from its color (RGB) and depth (infrared) cameras respectively

For the blur function, a separable Gaussian blur has been implemented. Given that a considerable amount of blur is needed, the convolution kernel has to be big. For a discrete kernel of a $2 \sigma$ radius (where $\sigma$ is the standard deviation of the Gaussian kernel), the loss of precision on peripheral values, may cause a diminution of luminosity. This effect is compensated dividing each element of the kernel by an empirically obtained constant ( 1.8 for this implementation).

For frame rectification, Microsoft's Kinect SDK 1.8 provides the MapDepthFrameToColorFrame() method which, as the name suggests, allows us to map depth pixels to their corresponding locations in color space.

The application's graphic interface displays a real time preview of the color camera view, a slider that allows the user to select the focal plane distance and a text box to select the $\sigma$ parameter for the Gaussian Blur kernel generation, that is, the blur radius (directly proportional to its intensity). 
Equation 2 with parameters $d_{f}$ determined by the user at execution time and $\sigma=$ 0.3 has been selected as the pixel-weight function.

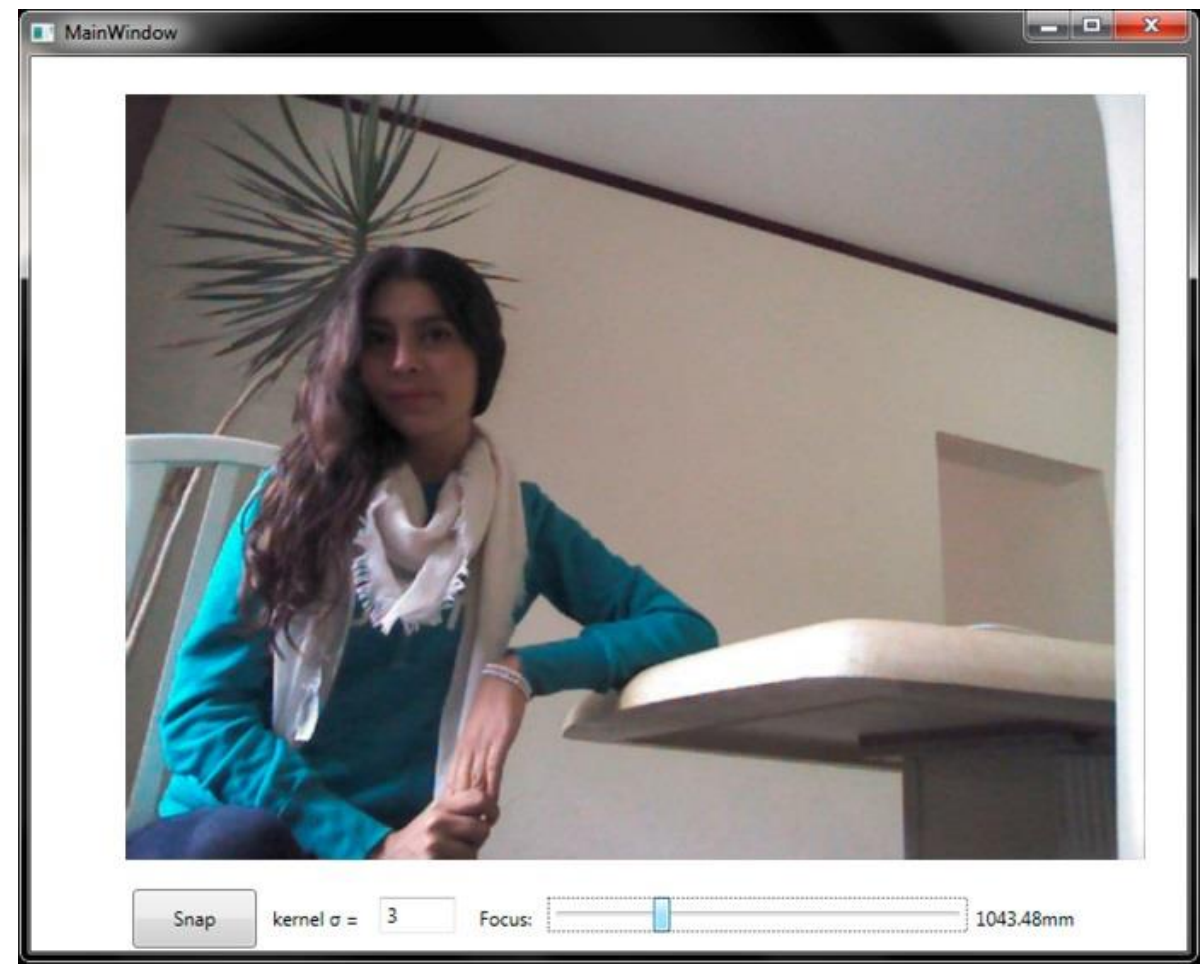

Figure 5. The application GUI

\section{Testing Methodologies}

In order to verify the effectiveness of the proposed process two tests were performed:

\subsection{Standalone test}

A single set of pictures were taken from a subject with natural lighting using the Microsoft Kinect sensor with color and depth resolutions of $640 \times 480$. The $\sigma$ parameter (standard deviation) of the Gaussian blur kernel was set to 4 and focal plane distance at $1510 \mathrm{~mm}$ (33.5\% of the max range). Figure 6 portrays the results of this test. 


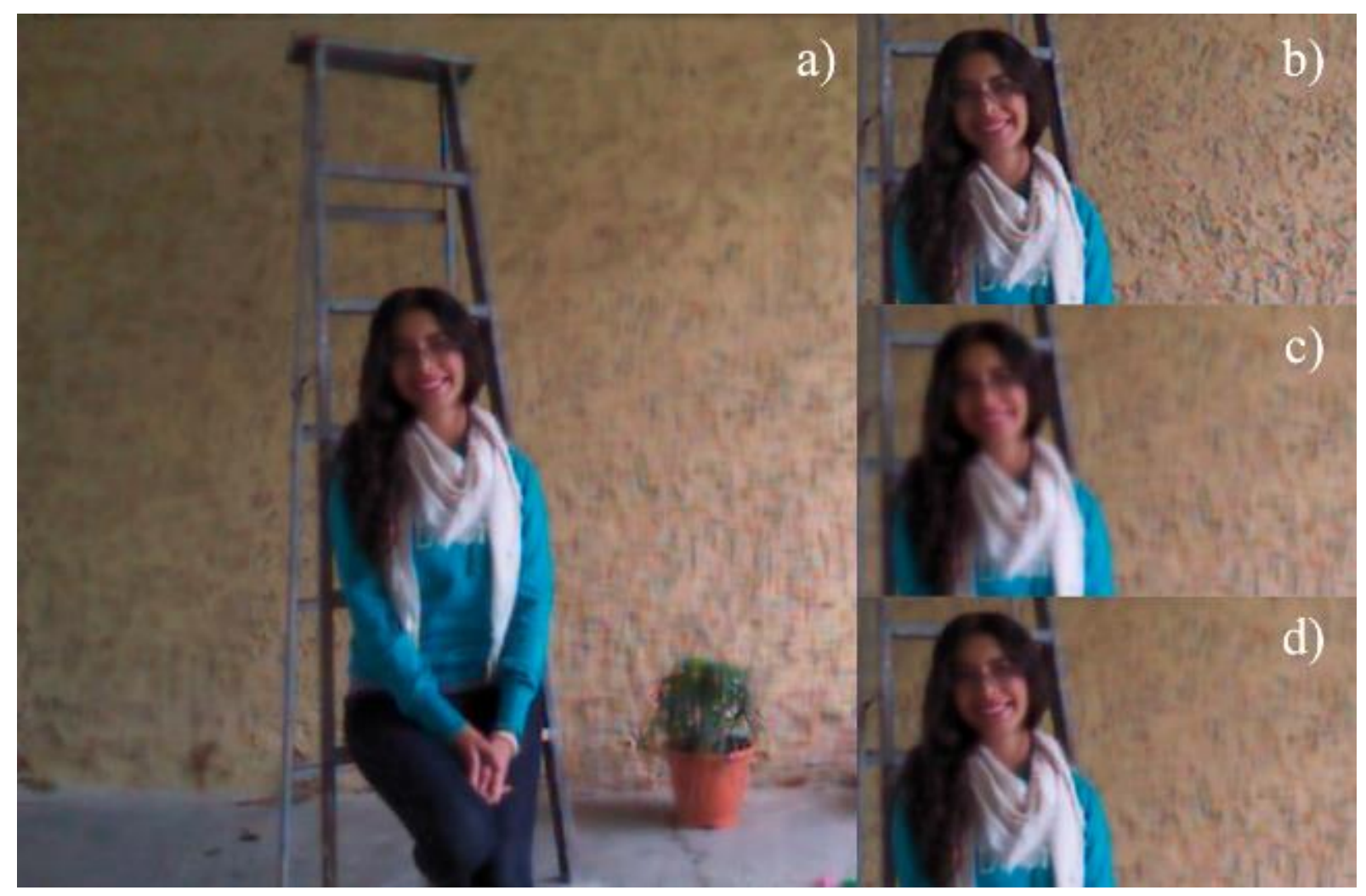

Figure 6. Results from the standalone test: a) Final image. b) Detail from the original image. c) Detail from the blurred copy. d) Detail from the final image.

\subsection{Benchmark test}

To perform a qualitative analysis of the final images rendered with this technique, its results were compared to similar shots obtained with a regular consumer digital camera. Reference pictures were taken with a Canon REBEL EOS T5i camera with an aperture of $f / 4$ at $1 / 80$ s and a color temperature of approximately $4000 \mathrm{~K}$ to achieve similar colors to those from the Kinect. Both the reference camera and the Kinect where placed at the same distance from the subject, that is, $1.40 \mathrm{~m}$ (see figure 7). For comparison purposes the Kinect image was horizontally mirrored from its original orientation. Color and depth resolutions were set to $640 \times 480$. 


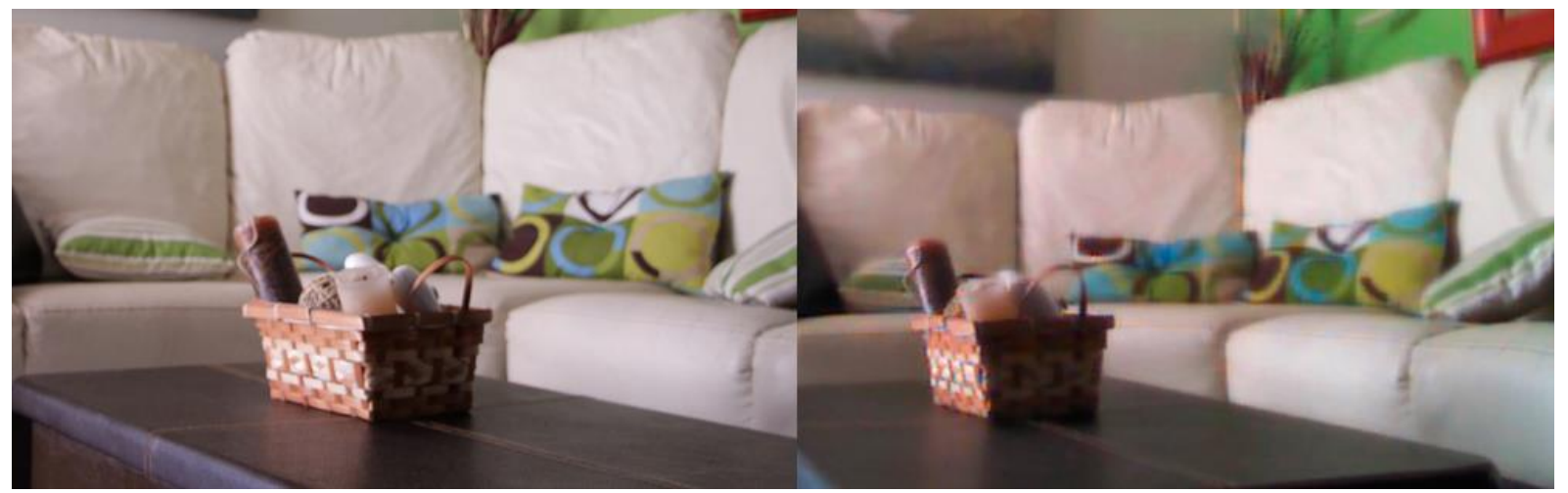

Figure 7. Results from the benchmark test: Consumer digital camera (left) and Microsoft Kinect (right).

\section{Results}

As depicted in figure 6 , the pictures provided by the 3D camera using the proposed Depth of Field Simulation algorithm yielded natural-looking results. Images from the benchmark testing show a similar quantity and quality of blur in out-of-focus areas, suggesting that the simulation algorithm paired with a depth camera constitutes a good substitution candidate for traditional camera lenses in small devices.

It is important to point out that some inaccuracies are present in both standalone and benchmark tests. These flaws are usually rendered around borders and reflecting surfaces and are a consequence of the infrared-based sensor limitations.

\section{Conclusions and future work}

Throughout this work the usage of 3D cameras to simulate the depth of field proved to be a promising methodology to drastically improve the quality of the 
pictures without the need for expensive, heavy and delicate additional optics. This is particularly interesting if, as stated by some vendors, depth sensors become cheaper, smaller and widely available in coming years.

This method differs from others currently on the market solely on the acquisition time of the depth map which for Microsoft Kinect and Creative Senz3d can be as fast as $30 \mathrm{fps}$ seconds (around 0.0333 seconds) and up to $60 \mathrm{fps}$ (.0166 seconds) for Occipital Structure sensor, a process that might take several seconds for current implementations of optical-based methods such as the Google Camera app.

This paper offers as well interesting areas of improvement, such as the use of additional depth measurement techniques (like optical or acoustic sensors) to increase the accuracy and quality of the images or the utilization of Graphics hardware to speed up the processing of the highly parallelizable operations taking place in the current implementation, making possible its usage for realtime video capturing as well.

\section{References}

Alkouh, H. B. (2002). U.S. Patent No. 7081892.

Buhler, J., \& Dan, W. (2002). A Phenomenological Model for Bokeh Rendering. ACM SIGGRAPH 2002 conference abstracts and applications (p. 142). New York, NY, USA: ACM.

Hernández, C. (2014, April 16). Lens Blur in the new Google Camera app. Retrieved from Google reasearch blog: http://googleresearch.blogspot.mx/2014/04/lens-blur-in-new-google-cameraapp.html

Ng, R. (2006). Digital light field photography. (Doctoral Dissertation). Stanford University.

Rigger, G., Tatarchuk, N., \& Isidoro, J. (2003). ShaderX2 - Shader Programming Tips and Tricks with DirectX 9. Wordware. 
Tibken, S. (2013, November 29). Wave fingers, make faces: The future of computing at Intel. Retrieved from Cnet news:

http://www.cnet.com/news/wave-fingers-make-faces-the-future-ofcomputing-at-intel/

Wu, J., Zheng, C., Hu, X., Wang, Y., \& Zhang, L. (2010, June 1). Realistic rendering of bokeh effect based on optical aberrations. The Visual Computer, 26(6-8), 555563.

Z, E. (2014, April 14). tempo.co. Retrieved from http://en.tempo.co/read/news/2014/04/18/240571603/Google-ReleasesAndroid-Camera-App

\section{Biographical notes:}

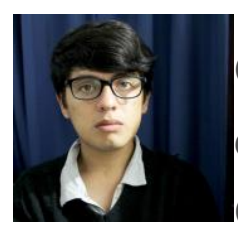

Omar Alejandro Rodríguez Rosas He obtained his Bachelor's degree in Computer Science Engineering from the University of Guadalajara. He worked for two years at Intel as an Intern for the Visual and Parallel-Computing Group. He is currently a member of the multidisciplinary art collective Proyecto Caos and collaborates as a research assistant for the Intelligent Systems laboratory at the University of Guadalajara's University Center for Exact Sciences and Engineering.

\section{(c) (1)(2)}

Esta obra está bajo una licencia de Creative Commons Reconocimiento-NoComercial-Compartirlgual 2.5 México. 